\title{
An empirical comparison of calibration and validation methodologies for airborne imaging spectroscopy
}

\author{
K. N. Babu ${ }^{1, *}$, A. K. Mathur ${ }^{1}$, David R. Thompson ${ }^{2}$, Robert O. Green ${ }^{2}$, \\ Piyushkumar N. Patel $^{3}$, R. P. Prajapati ${ }^{1}$, Brian D. Bue ${ }^{2}$, Sven Geier ${ }^{2}$, \\ Michael L. Eastwood ${ }^{2}$ and Mark C. Helmlinger ${ }^{2}$ \\ ${ }^{1}$ Space Applications Centre (ISRO), Ahmedabad 380 015, India \\ ${ }^{2}$ Jet Propulsion Laboratory, California Institute of Technology, USA \\ ${ }^{3}$ Physical Research Laboratory, Ahmedabad 380 009, India
}

To date, a large number of existing applications in India have used multi-band observations from airborne and spaceborne platforms. New sensors are providing additional capabilities thanks to special aerial missions with the compact airborne spectrographic imager (CASI), the short-wave infrared (SWIR) full spectrum imager (SFSI) and the National Aeronautics and Space Administration's (NASA's) Next Generation Airborne Visible/Infrared Imaging Spectrometer (AVIRIS-NG). Opportunities to exploit quantitative spectroscopic signatures and high spatial resolution have garnered great interest among the scientific community, and the success of these missions will rely on accurate calibration. Here we focus on a vicarious calibration experiment conducted for the AVIRIS-NG India campaign. We discuss initial validation results, with descriptions of in situ and remote calibration and measurement protocols, geometric processing with precise position and attitude data, and atmospheric simulations used to validate the remote measurement. A partnership between Indian Space Research Organisation (ISRO) and NASA investigators proved a unique opportunity to assess the empirical variability in results, indicating their sensitivity to modelling choices and assumptions. The vicarious calibration exercise uses multiple radiative transfer models, including MODTRAN 6.0 and a new version of the $6 \mathrm{~S}$ radiative transfer code, viz. 6SV2.1, which is capable of accounting for polarization.

Keywords: Hyperspectral measurements, radiative transfer, reflectance, vicarious calibration.

\section{Introduction}

A portion of this research was carried out at the Jet Propulsion Laboratory, California Institute of Technology, Pasadena, USA under a contract with the National Aero-

*For correspondence. (e-mail: kn_babu@sac.isro.gov.in)

nautics and Space Administration (NASA). The Next Generation Airborne Visible/Infrared Imaging Spectrometer (AVIRIS-NG) instrument and India campaign were sponsored by the NASA Earth Science Division. The US Government Support is Acknowledged.

As the Earth's population grows and prospers economically, it has an increasing impact on the eco-system and environment. Left unchecked, this development could threaten common natural resources and risk additional pollution of the Earth's land surface, freshwater, coastal areas and oceans. Regular monitoring and accounting of these resources is essential for their effective usage and management. Though there are many tools and techniques for this purpose, remote sensing stands out as a recent and significant contributor. Remote sensing allows users to collect data over wide spatial and temporal domains on local to global scales. Such comprehensive measurements would be difficult, if not impossible, from ground-based experiments. Though it has many advantages, robustness of remote sensing depends heavily on the accuracy of the measurement system and the process for inferring the remote geophysical parameters of interest. To be useful for resource monitoring, the scientific community needs to know if the changes in retrieved environmental measurements are in fact real, or whether they are due to changes in the sensor or the calibration system used to monitor these sensors. Accurate calibration is even more crucial for studies requiring long-term trend analysis. Accurate retrieval of bio-geophysical parameters from such sensors demands careful attention to radiometric calibration and its verification ${ }^{1-3}$. Ultimately, the reliability of the absolute radiometric accuracy is directly related to the accuracy of this calibration. Without accuracy of calibration and validation exercises, uncertainty would propagate to downstream analyses and any environmental policy that results from them.

Vicarious calibration can enable highly reliable in-flight radiometric calibration using in situ measurements of both accurate surface radiance/reflectance and atmospheric 
parameters ${ }^{4}$. This is an absolute calibration method that yields a new set of radiometric coefficients which can be used as an alternative to those derived in the laboratory ${ }^{2,5}$. Alternatively, such experiments can validate the initial calibration and track any trends over time. However, vicarious calibration involves a more complicated optical system than the laboratory environment. The aerosol optical depth (AOD) and surface reflectance retrievals are not independent; one factor may increase or decrease the uncertainty of the other. For example, a change in the surface reflectance could either increase or decrease the atmospheric aerosol contribution of the apparent radiance and vice versa, depending on the spectral range and optical properties of the scattering particles. This interdependence is most significant for dark targets, where the additive signal from atmospheric aerosol and Rayleigh scattering is strongest relative to the reflected solar illumination. Conversely, a relatively small variation in AOD would not change the apparent radiance significantly when surface reflectance is high. Such interdependencies require accurate knowledge of both the quantities.

Recently, a joint effort between the Indian Space Research Organisation (ISRO) and the National Aeronautics and Space Administration (NASA), USA provided impetus for a cooperative imaging spectroscopy campaign over diverse targets in India with NASA's AVIRIS-NG. This campaign deployed the instrument from December 2015 to March 2016 and January to May 2018, visiting diverse biomes, elevations and locations across the Indian subcontinent. The major advantage of imaging spectroscopy - also known as hyperspectral imaging - for remote sensing is the ability to measure spectral radiance in many narrow spectral bands across wide geographic areas. In other words, the instrument can acquire the full radiance spectrum at every pixel within an image, enabling quantitative spectroscopic mapping of geophysical phenomena. The 'Classic' AVIRIS sensor acquired contiguous spectral measurements between 380 and $2510 \mathrm{~nm}$ at $10 \mathrm{~nm}$ spectral sampling for over two decades ${ }^{6}$. More recently, AVIRIS-NG has been deployed for many campaigns; it measures a similar spectral range at $5 \mathrm{~nm}$ with improved signal-to-noise ratios (SNRs) ${ }^{7}$.

This article details a vicarious calibration methodology applied to AVIRIS-NG data during the campaign. Our goal is to give the reader an understanding of the influence of vicarious calibration methods and experimental decisions on the results, and of the accuracy of AVIRISNG data which can be expected for downstream analysis. We also used the joint campaign to explore how differences in modelling methods and radiative transfer assumptions can affect the result. While airborne imaging spectrometers consistently strive for high accuracy, many incidental assumptions in this process can introduce variability at the per cent level. In most cases, multiple choices are defensible. Such choices include the methods for excluding outliers, particular areas used for spatial averaging, atmospheric assumptions in radiative transfer and other properties that cannot be measured directly, such as aerosol scattering asymmetry. The India investigation involved scientists from multiple organizations, providing an excellent opportunity to evaluate the impact of these unknowns. To that end, partner teams from ISRO and Jet Propulsion Laboratory (JPL) used two independent simulation methods to predict radiance, namely MODerate resolution atmospheric TRANsmission radiative transfer code (MODTRAN 6.0) ${ }^{8}$ and a new version of the $6 \mathrm{~S}$, second simulation of a satellite signal in the solar spectrum, radiative transfer code (6SV2.1), which has a module to account for the polarization component of radiation ${ }^{9}$. This provides independent assessments of the AVIRIS-NG calibration during the campaign.

\section{Measurement methodology}

The study area, Desalpar, comprises parts of the Rann of Kutch, Gujarat, India, which is an extensive plain of salty marsh desert in the Kutch district of western Gujarat (Figure 1). The site is basically a dry, salty terrain, semiarid region with high temperatures and high surface reflectance during February-May. Whereas during June-January this area is prone to extensive waterlogging because of rainfall as well as water ingress due to sea and river ${ }^{10}$.

The site was selected for beneficial atmospheric properties and the presence of a large, uniform, bright unvegetated surface. Both surface and atmospheric parameters vary over time; so the measurements were designed to be conducted simultaneously with the overhead flight schedule. Even if surface physical properties did not change significantly on hourly scales, changes in solar illumination would induce variable reflectances due to the influence of bidirectional reflectance properties of the surface ${ }^{10}$. We aimed to estimate the hemispherical directed reflectance function (HRDF), which is the apparent surface reflectance from the entire sky illumination, including diffuse and directed solar illumination, as measured in the direction of the AVIRIS-NG sensor. This quantity is time-dependent due to self-shading by microtexture and other bi-directional reflectance distribution function (BRDF)-related physical properties of the surface. Similarly, AOD would change rapidly over time due to effects like wind-lofted dust or natural variability in the background aerosol conditions at the site. For these reasons, coincident observations are essential.

Field measurement campaigns were carried out on 9 and 10 February 2016. The measurements of bidirectional surface reflection were done using a field-portable analytical spectral device (ASD) spectroradiometer, which measured reflected radiance from 350 to $2500 \mathrm{~nm}$. The instrument details can be found in www.asdi.com. Measurements of surface reflectance were carried out in a well-defined pattern in the field. The instrument used a 
sensor head with $25^{\circ}$ full field of view bare optics attached to a monopod stick and held away from the user's body. The stick-mounted optics eliminated the possibility of viewing any self-shade by the instrument operator. The team calculated average reflectance of the surface by ratioing measured radiances with those of a bright reference panel, the reflectance of which was known in advance. These 'white reference' measurements were taken every $5 \mathrm{~min}$ during the campaign. Any atmospheric changes over these short intervals would affect both reference and terrain targets equally; so the ratio cancelled them out ensuring temporal consistency. The surface reflectance measurements covered four rectangular quadrants forming a larger rectangle, approximately $20 \times 10$ pixels in size. Figure 2 shows the surface reflectance spectra mean (solid red line), standard deviation (error bar) and percentage of uncertainty in the measurements. The uncertainty in the mean assumes noise is zero-mean and Gaussian; this variability is due to both instrument noise as well as surface homogeneity. The team acquired two sets of surface measurements at 11:30 $\mathrm{h}$ and 12:00 $\mathrm{h}$ local time, with 133 and 136 surface reflectance spectra respectively, on the actual day of AVIRIS-NG pass, i.e. 10 February 2016.

Photons traversing the atmosphere were also influenced by the presence of aerosols, integrated water vapour (WV) and other constituents. The team made measurements

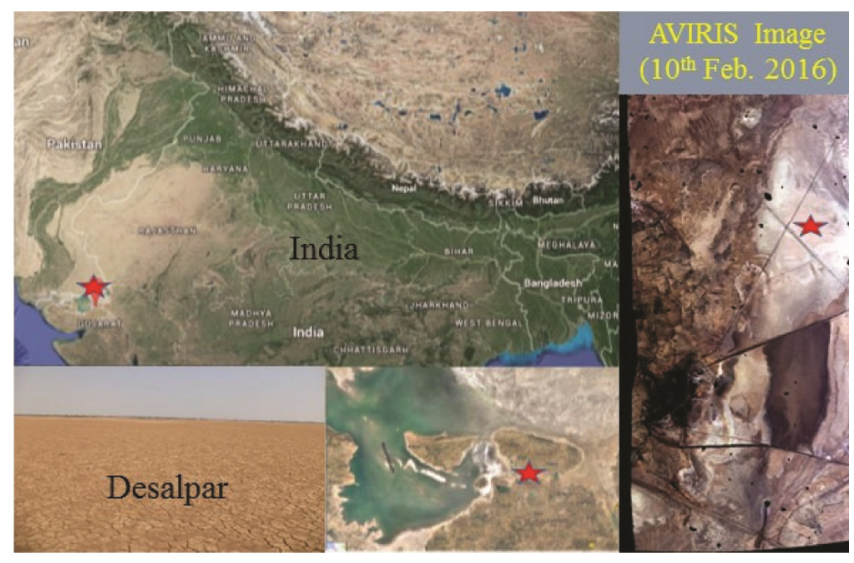

Figure 1. The calibration site at Desalpar, Gujarat, India.

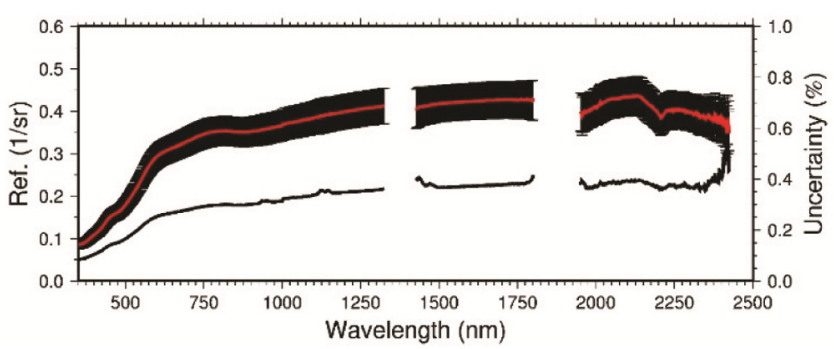

Figure 2. Surface reflectance using ASD spectrometer (red) and its uncertainty (lower black line). The team acquired the measurement at 12:00 h local time, with 136 distinct spectral samples (black envelope around the red line). of columnar spectral AOD, WV and ozone content with a well-calibrated Microtops-II sun-photometer and Ozonometer (the calibration was performed in advance at a ground-instrumented site, viz. Mount-Abu, during October 2015); more details about the instrument can be found at www.solarlight.com. During the Desalpar overhead flight pass, the Microtops-II measurements were acquired at $15 \mathrm{~min}$ interval. Figure 3 presents the mean value and standard deviation of AOD, WV and ozone. The scaling of WV and ozone are mentioned in centimetres in view of the fact that total perceptible water and ozone content above the measurement height are expressed in equivalent to centimetres height in most of the atmospheric radiative transfer models. Note that the atmosphere was pristine with a mean AOD value of 0.116 at $870 \mathrm{~nm}$ during this field campaign. The timing of the calibration campaign provided an excellent opportunity to study the AVIRIS-NG performance over this site, since it fell in mid-winter when the surface was mostly dry and there was a clear atmosphere with less aerosol loading compared to the spring or summer seasons ${ }^{11}$. Earlier studies had characterized the monthly variability in AOD over the Desalpar site ${ }^{11}$. AOD showed a distinct annual pattern with a decreasing trend from autumn to winter and then continuous increase during the spring and summer period, attaining a maximum value in July.

AVIRIS-NG acquired four aerial survey lines over the calibration site during the December 2015-March 2016 phase of the campaign. We performed vicarious calibration with the two coincident overhead measurements having IDs 'ang20160210t061239' and 'ang20160210 t062002'. Radiance data were initially calculated at JPL using laboratory calibration methods ${ }^{12}$. These experiments characterized the radiometric and spectral response properties of the AVIRIS-NG instrument, including any sub-percent variability over the focal plane array (FPA). Atmospheric features were then used to fit and correct for any sub-pixel drift in spectral calibration over time, and for any non-Gaussian imperfections in instrument spectral response. Previous studies had shown the typical cross-track variability in spectral response of AVIRISNG as determined by the position of the oxygen absorption feature at $760 \mathrm{~nm}$, which was typically less than $2 \%$ of channel width ${ }^{13}$. The initial laboratory radiometric

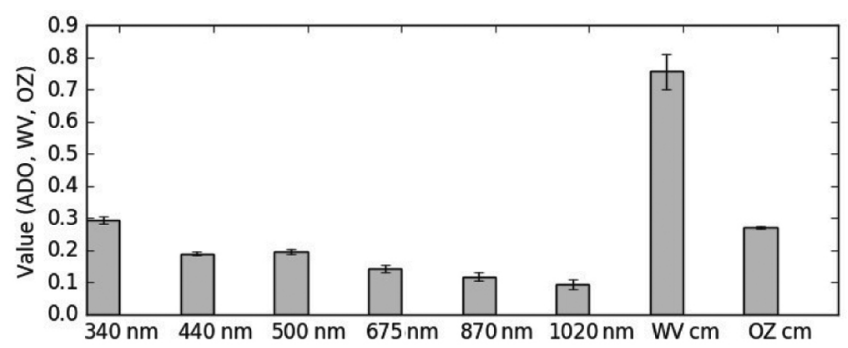

Figure 3. Microtops-II derived values of aerosol optical depth (AOD), water vapour $(\mathrm{WV}, \mathrm{cm})$ and ozone content $(\mathrm{OZ}, \mathrm{cm})$. 


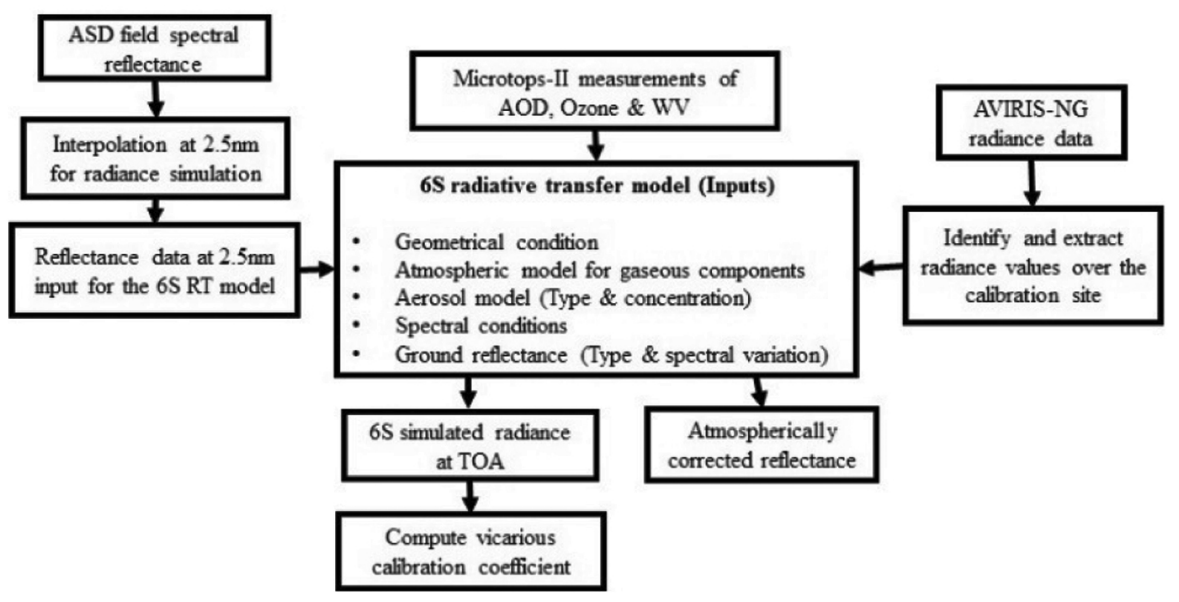

Figure 4. Model flowchart for the top of the atmosphere radiance simulation.

calibration used a calibrated SI-traceable illumination source imaged under controlled geometry. Subsequent validation experiments performed at Ivanpah Playa (California, USA) demonstrated that the procedure has the potential to achieve calibration accuracy within nominal specifications, better than $5 \%$ under optimal conditions and occasionally achieving accuracies within $2 \%$. For the India campaign, the instrument team updated the initial result with multiple field calibrations using a moving integrating sphere in illumination-controlled hangar conditions. These were used to update the flat field (i.e. FPA element-specific responses), resulting in three calibration solutions for different segments of the multi-month campaign.

In addition to the radiometric and spectral calibration, AVIRIS-NG used a geometric calibration based on a model of view angles of independent FPA elements. The instrument team fitted a geometric camera model before the campaign by identifying manual ground control points of a known location within an imaged scene, and then optimizing the model parameters with bundle adjustment to match the known coordinates. During the campaign, the team orthorectified new scenes by combining the geometric camera model with integrated inertial measurement system (IMS) and global positioning system (GPS) measurements. The IMS/GPS data allowed highly accurate sensor position estimation. Then, for each spectrum, the optical path from the appropriate FPA element was traced through the camera model down to a digital elevation model of the surface to determine the specific location observed by each instrument integration timestep. The team then georectified the spectral data cubes, projecting them with nearest-neighbour interpolation onto a rectangular geo-referenced grid for comparison with the ground measurements. The standard AVIRIS-NG radiance products, also known as 'Level 1b' data products, contained orthorectified data as well as other metadata such as solar geometry and surface elevation.

\section{Vicarious calibration}

The vicarious calibration methodology follows a 'reflectance-based' approach that is widely used for accurate calibration of remote sensing instruments ${ }^{14,15}$. Other popular alternatives include radiance and irradiance methods. The present reflectance-based method used in situ data to predict the remotely measured radiance, and then compared it to the airborne measurement. As mentioned, two separate teams worked in partnership on different simulations. Each involved different 'radiative transfer models' of physical photon transport in the atmosphere. This gave insight into the empirical variability of results induced by legitimate differences in modelling assumptions, and provided extra confidence in any validation result.

The first simulation used the second simulation of a satellite signal in the solar spectrum version 2.1 (hereafter 6Sv2.1) polarized version of the atmospheric radiative transfer model to calculate the at-sensor radiance over the test site. Figure 4 is a flowchart of the methodology used for the 6Sv2.1 simulation. Microtops-II atmospheric values for ozone, WV and AOD were adopted directly as inputs into the radiative transfer simulation. The team then simulated at-sensor radiance using the mean surface reflectance spectrum, calculating the result at $2.5 \mathrm{~nm}$ spectral sampling for both the reflectance datasets.

Some parameters could not be measured directly and were instead inferred from climatology. For unmeasured trace gases, the $6 \mathrm{~Sv} 2.1$ simulation adopted abundance profiles from the US62 atmospheric model. Temperature and pressure levels were also specified with this standard. The field campaign did not directly measure the physical and chemical nature of the aerosols. Given that the test site was not affected by the warm and humid air, the 6Sv2.1 team used an urban aerosol model with four different components. User definition options in the $6 \mathrm{~S}$ model (6S manual) were utilized to select the fractional abundance of different aerosol components. The team 
Table 1. Parameters used for two Desalpar atmospheric radiative transfer models

\begin{tabular}{lll}
\hline Parameter & \multicolumn{1}{c}{ Value } & \multicolumn{1}{c}{ Source } \\
\hline 6Sv2.1 & \multicolumn{1}{c}{ In situ data } \\
Pressure altitude & $0.75 \mathrm{~cm}$ & Microtops-II \\
Precipitable $\mathrm{H}_{2} \mathrm{O}$ vapour & $0.3-0.1(300-870 \mathrm{~nm})$ & Microtops-II \\
Total aerosol optical depth (AOD) & User-defined mixture & Climatology \\
Aerosol optical properties & $0.28 \mathrm{~cm}$ & Microtops-II \\
Atmospheric ozone & Variable & ASD spectrometer \\
Surface reflectance & & \\
& & \\
MODTRAN 6.0 & $13 \mathrm{~m}$ & In situ data \\
Pressure altitude & $1.1 \mathrm{~cm}$ & Match to spectra \\
Precipitable $\mathrm{H}_{2} \mathrm{O}$ vapour & $0.3-0.1(300-870 \mathrm{~nm})$ & Microtops-II \\
Total AOD & SSA $0.5-1.0(300-870 \mathrm{~nm})$ & Match to spectra \\
Aerosol optical properties & $300 \mathrm{DU}$ & Orbital data \\
Atmospheric ozone & Variable & ASD Spectrometer \\
Surface reflectance & & \\
\hline
\end{tabular}

defined aerosol component fractions of $0.1,0.55,0.0$ and 0.35 for dust-like, water-soluble, oceanic and soot aerosols respectively, through iterating the $6 \mathrm{~S}$ model for its spectral radiance profile against the AVIRIS-NG radiance.

The second simulation team used the MODTRAN 6.0 radiative transfer code with tropical atmospheric pressure, temperature and abundance profiles ${ }^{14}$. It calculated solar-reflected scattering and gaseous absorption using the DISORT radiative transfer engine, and correlated- $k$ methods to account for the finite channel widths. Viewing geometry, solar geometry and AOD were equivalent to the $6 \mathrm{~Sv} 2.1$ case. However, there were several important differences in modelling assumptions. First, the MODTRAN simulation did not include polarization. Second, the JPL team permitted small adjustments to the water vapour column which were diverged from the Microtops-II values. This accounted for the fact that the airborne instrument did not view absorption along the same optical path as the Microtops-II sun-photometer. The former observed water absorption along the aircraft/ ground/sun path, while the latter only observed the upward slant path to the sun. These differences could have translated into significant discrepancies in the estimated tropospheric WV column amounts due to significant spatial variability in WV. Additionally, the AVIRIS-NG sensor provided its own highly accurate estimate of WV by measuring the precise shape of multiple absorption features with tens of measurements each. For these reasons, the JPL team favoured a WV measurement from the remote sensor; they adjusted the model to achieve the best radiance matches within these absorption features. The resulting value differed by about $0.35 \mathrm{~cm}$ from the Microtops-II estimate (Table 1).

The aerosol parameterization also differed from the $6 \mathrm{~S}$ simulation. The MODTRAN RTM had the ability to incorporate the channellized wavelength-dependent aerosol extinction information from the Microtops-II directly. Single scattering albedos ranging from 0.5 to 1.0 , consis- tent with urban absorbing aerosols, adjusted within reasonable ranges defined for each wavelength. A physically plausible asymmetry parameter of 0.65 was used.

\section{Results and discussion}

There are several ways to use the results of a validation exercise. Validating the instrument calibration can be as simple as comparing the remote measurement to the prediction, and calculating a ratio of the two ${ }^{1,16,17}$. This ratio can then be applied as a radiance correction factor in subsequent data products from the campaign. Alternatively, the in situ measurement uncertainties can be incorporated into a holistic uncertainty budget in order to validate the calibration, if the uncertainty model can fully explain any departures. An example of the latter approach can be found in Thompson et al. ${ }^{13}$. In our experiment, we began by comparing the predicted and actual radiances; Figures 5 and 6 provide examples from the two simulation methods, i.e. 6Sv2.1 and MODTRAN 6.0 respectively. The 6Sv2.1 simulation analysed two sets of ASD data, plotted separately. Each chart compares the remote measurement (in red) to the prediction (in blue), with the residual difference as a black line. Indeed, the differences between the two simulations lead to variability that is at least as large as the residuals themselves.

Overall the agreement between model predictions and results was good, achieving better than 5\% absolute agreement and superior relative agreement in shapes. One departure outside this regime was the extreme blue spectrum near $400 \mathrm{~nm}$, where both models showed differences above $0.5 \mu \mathrm{W} \mathrm{nm} \mathrm{cm}^{-1} \mathrm{sr}^{-1}$. This is not unusual, since aerosol scattering and variability in this spectral range are difficult to measure or predict, and would significantly affect the result.

Identifying the inevitable discrepancies that do result from any vicarious calibration experiment involves 
examining the uncertainties in all input data products, and in those model unknowns which could not be measured or retrieved directly. Similarly, any small uncertainty in direct atmospheric measurements, variability in surface reflectance, some other combination of unknowns (such as unknown atmospheric properties), or instrument calibration can be larger sources of any discrepancies. During this calibration campaign the surface reflectance observations were acquired from $11: 00$ to $12: 05 \mathrm{~h}$ (local time), during which the solar zenith angle varied from $55^{\circ}$ to $40^{\circ}$. The AOD at $500 \mathrm{~nm}$ varied from 0.183 to 0.22 during this campaign, indicating a clear atmosphere. The derived integrated WV and column ozone ranged from 0.66 to $0.87 \mathrm{~cm}$ and 251 to $276 \mathrm{DU}$ respectively. The clear and dry atmosphere was attributed to the winter season when northeasterly winds carried away most of the aerosol and moisture. The uncertainty in AOD at $500 \mathrm{~nm}$ channel was close to $0.2 \%$ with standard deviation of $0.88 \%$ (Table 2). The WV uncertainty from the Microtops-II was $0.68 \%$ with standard deviation of $5.5 \%$. The ozone content gave an uncertainty of $0.052 \%$, with percentage of standard deviation of 0.43 . The spectral uncertainty in surface reflectance measurements varied from $0.2 \%$ to $0.4 \%$; thus the surface was spectrally flat and
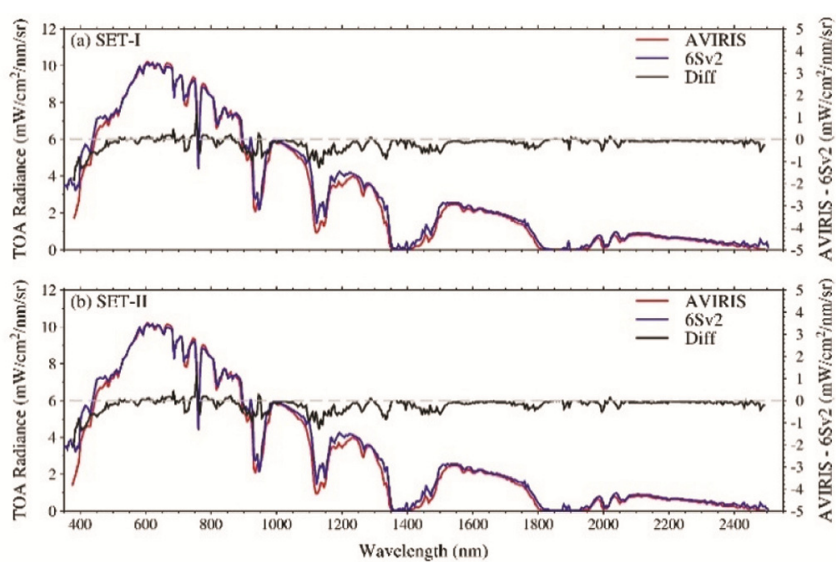

Figure 5. Comparison of simulated and AVIRIS-NG measured radiance over Desalpar using the $6 \mathrm{~Sv} 2$ model.

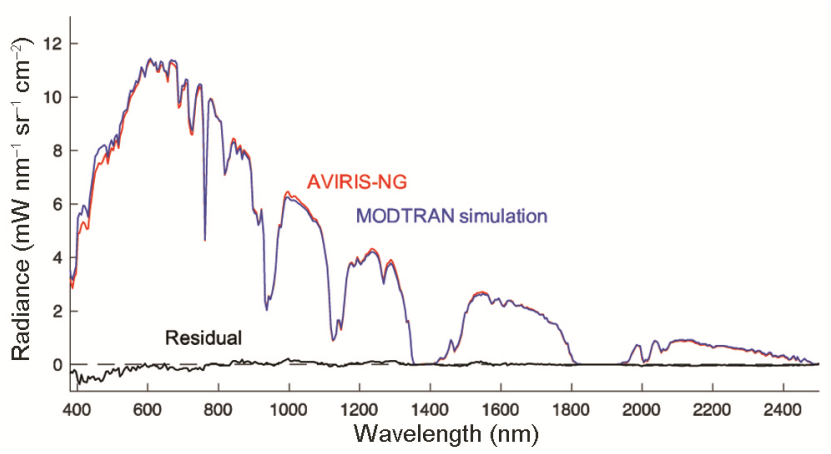

Figure 6. Comparison of simulated and AVIRIS-NG measured radiance over Desalpar using the MODTRAN 6.0 model. hence qualified as a good calibration site for highresolution aerial sensors such as AVIRIS-NG.

Column profile temperatures and pressures are a large source of this uncertainty, since neither the US62 nor tropical profiles were a perfect match to the conditions on site. To the degree that future field teams can measure profile values directly, with radiosondes or ground-based sounding instrumentation, this could assist future campaigns in reducing these uncertainties. Subtle differences in aerosol scattering properties from modelled assumptions also affected the scattering profile, which likely had large influence on short wavelengths. Aerosol optical property knowledge is more important for spectrometers than many other multi-band methods, since the spectroscopic observation is highly sensitive to curvature of the radiance profile in this spectral range. Custom climatologies derived from dedicated orbital instruments or longterm study of calibration sites could help in reducing this uncertainty. Finally, the AVIRIS-NG calibration is also least certain in the blue range due to the challenge of achieving adequate illumination in the blue/UV portion of the spectrum ${ }^{18}$. Typical calibration lamps have a colour temperature of $3000 \mathrm{~K}$, while the solar spectrum is closer to $6000 \mathrm{~K}$. This results in a paucity of photons in the short-wavelength range during calibration, and may have contributed in part to the systematic discrepancy at $400 \mathrm{~nm}$.

The ultimate reliability of a calibration prediction is often described by a radiometric calibration uncertainty, reported as a standard deviation in each channel. The ISRO team calculated this value from the $6 \mathrm{~Sv} 2.1$ simulation results, estimating from a composite of the separate factors that contribute to the overall uncertainty. These factors were assumed to be independent of each other, such that the overall uncertainty was the root sum of squares of the constituent uncertainties ${ }^{11,19}$. The correlation coefficient between the model simulation and AVIRIS-NG L1B radiances was $0.995,0.995$ and RMSE was $0.36,0.37 \mathrm{~mW} / \mathrm{cm}^{2} / \mathrm{nm} / \mathrm{sr}$ for set-1 and set-2 respectively. To determine the uncertainty in TOA simulation due to variability in surface reflectance, additional model runs were made by perturbing the reflectance by one standard deviation in each direction. The uncertainty values using these three simulations showed $15 \%$ averaged value over the spectral range between 400 and $1200 \mathrm{~nm}$. For the full range, the average uncertainty was $8.6 \%$. Such differences were typically larger than the observed discrepancy between in situ and remote data. Consequently, spatial variability in surface reflectance could be another major contributor to the differences between predicted and actual remote measurements.

\section{Conclusion}

Collaboration between NASA and ISRO led to a vicarious calibration and modelling experiment to determine 
Table 2. Uncertainty in the atmospheric component measurements

\begin{tabular}{lccccccc}
\hline Parameter & AOT-340 & AOT-440 & AOT-500 & AOT-675 & AOT-870 & WV (cm) & Oz (cm) \\
\hline Uncertainty (\%)* & 0.222 & 0.175 & 0.211 & 0.230 & 0.259 & 0.68 & 0.052 \\
Standard deviation (\%) & 1.08 & 0.67 & 0.88 & 1.05 & 1.33 & 5.5 & 0.43 \\
\hline
\end{tabular}

*Uncertainty $=$ Standard deviation $/(N)^{1 / 2}$.

the performance of AVIRIS-NG sensor during its aerial campaign over the Indian sub-continent. The Desalpar playa, a desert site, was chosen for the post-launch vicarious calibration campaign due to beneficial illumination, surface and atmospheric conditions. The methodology used field measurements and radiative transfer simulations with two alternative modelling methodologies: The polarized version of the $6 \mathrm{~S}$ radiative transfer model, and the MODTRAN 6.0 radiative transfer model. In situ measurements of atmosphere and surface reflectance were incorporated and used to predict the at-sensor radiance. These predictions validated the AVIRIS-NG calibration. Unknowns in the radiative transfer modelling and differences between the two simulation methodologies were larger than the observed discrepancy with the remote measurement. This favoured keeping the laboratory calibration solution for AVIRIS-NG, and underscored the importance of more detailed atmospheric measurements in the future to drive-down remaining uncertainties and permit ever more accurate vicarious calibration. Overall, this experiment demonstrated a successful collaboration and partnership between NASA and ISRO, laying a promising foundation for joint calibration exercises in the future.

1. Naughton, D. et al., Absolute radiometric calibration of the rapid eye multispectral imager using the reflectance-based vicarious calibration method. J. Appl. Remote Sensing, 2011, 5(1), 053544; https://doi.org/10.1117/1.3613950.

2. Slater, P. N. et al., Reflectance-based and radiance-based methods for the in-flight absolute calibration of multispectral sensors. Remote Sensing Environ., 1987, 22, 11-37.

3. Thome, K. J., Absolute radiometric calibration of Landsat 7 ETM+ using the reflectance-based method. Remote Sensing Environ., 2001, 78, 27-38.

4. Thome, K. J., Arai, K., Tsuchida, S. and Biggar, S. F., Vicarious calibration of ASTER via the reflectance-based approach. IEEE Trans. Geosci. Remote Sensing, 2008, 46, 3285-3295.

5. Dinguirard, M. and Slater, P. N., Calibration of spacemultispectral imaging sensors: a review. Remote Sensing Environ., 1999, 68, 194-205.

6. Green, R. O., Eastwood, M. L. and Satrure, C. M., Imaging spectroscopy and the airborne visible/infrared imaging spectrometer (AVIRIS). Remote Sensing Environ., 1998, 65, 227-248.

7. Thompson, D. R., Natraj, V., Green, R. O., Helmlinger, M., Gao, B.-C. and Eastwood, M. L., Optimal estimation for imaging spectrometer atmospheric correction. Remote Sensing Environ., 2018, 216, 355-373.
8. Berk, A., Conforti, P., Kennett, R., Perkins, T., Hawes, F. and van den Bosch, J., MODTRAN6: a major upgrade of the MODTRAN radiative transfer codes. Proc. SPIE 9088, 2014, 7; doi:10.1117/ 12.2050433 .

9. Kotchenova, S. Y. and Vermote, E. F., Validation of a vector version of the $6 \mathrm{~S}$ radiative transfer code for atmospheric correction of satellite data. Part II: Homogeneous Lambertian and anisotropic surfaces, Appl. Opt., 2007, 46, 4455-4464.

10. Sridhar, V. N., Mehta, K. B., Prajapati, R. P., Babu, K. N., Suthar, N. M. and Shukla, A. K., Absolute vicarious calibration of OCM2 and AWiFS sensors using a reflectance based method over land sites in the Rann of Kutch, Gujarat. Int. J. Remote Sensing, 2013, 34, 5690-5708.

11. Patel, P. N., Dumka, U. C., Kaskaoutis, D. G., Babu, K. N. and Mathur, A. K., Optical and radiative properties of aerosols over Desalpar, a remote site in western India: source identification, modification processes and aerosol type discrimination. Sci. Total Environ., 2017, 575, 612-627.

12. Thompson, D. R., Boardman, J. W., Eastwood, M. L., Green, R. O., Haag, J. M. and Gorp, B. V., Imaging spectrometer stray spectral response: in-flight characterization, correction and validation. Remote Sensing Environ., 2018, 204, 850-860.

13. Thompson, D. R. et al., Real-time remote detection and measurement for airborne imaging spectroscopy: a case study with methane. Atmosph. Meas. Tech., 2015, 8, 4383-4397.

14. Pagnutti, M., Ryan, R. E., Kelly, M., Holekamp, K., Zanoni, V., Thome, K. and Schiller, S., Radiometric characterization of IKONOS multispectralimagery. Remote Sensing Environ., 2003, $\mathbf{8 8}, 53-68$.

15. Thome, K., Biggar, S. and Choi, H. J., Vicarious calibration of Terra ASTER, MISR, and MODIS. Proc. SPIE 5542, 2004; doi: $10.1117 / 12.559942$

16. Schott, J. R., Remote Sensing: The Image Chain Approach, Oxford University Press, 2007, p. 665.

17. Thome, K., Helder, D., Aaron, D. and Dewald, J., Landsat-5 TM and Landsat-7 ETM+ absolute radiometric calibration using the reflectance-based method. IEEE Trans. Geosci. Remote Sensing, 2004, 42, 2777-2785.

18. Helmlinger, M., Eastwood, M., Green, R. P. and Thompson, D. R., Solar-similar near-infrared suppressed 'blue' calibration source. In IEEE Aerospace Conference, Big Sky, USA, MT, 2016; doi:10.1109/AERO.2016.7500714.

19. Biggar, S. F., Slater, P. N. and Gellman, D. I., Uncertainties in the in flight calibration of sensors with reference to measured ground sites in the 0.4-1.1 $\mu \mathrm{m}$ range. Remote Sensing Environ., 1994, 48, $245-252$.

ACKNOWLEDGEMENTS. We thank the Director, SAC (ISRO, Ahmedabad) for encouragement and the Deputy Director, EPSA for valuable suggestions. We also thank the team members at the Desalpar Cal-Val site for help during data collection and the reviewers for their useful comments and suggestions.

doi: $10.18520 / \mathrm{cs} / \mathrm{v} 116 / \mathrm{i} 7 / 1101-1107$ 